\title{
Eficacia y seguridad a largo plazo de la estimulación del nervio vago: Reporte preliminar en pacientes adultos con epilepsia focal fármacorresistente.
}

Efficacy and safety of the long-term Vagus Nerve Stimulation: Preliminary report in adult patients with drug-resistant focal epilepsy.

Elliot Barreto-Acevedo ${ }^{1,2, a}$, Leila Barreto-Barra ${ }^{1, a}$, Mirla Villafuerte-Espinoza ${ }^{1,2}$, a, Alberto Díaz-Vásquez ${ }^{1, a}$, Alicia Becerra-Zegarra ${ }^{3, \mathrm{~b}}$, José Hernández-Vizarreta ${ }^{1, a}$, Victoria Llaja-Rojas ${ }^{1,4, c}$.

\section{RESUMEN}

Objetivo: Reportar la experiencia en eficacia y seguridad a largo plazo de la estimulación del nervio vago (ENV), en una serie de pacientes adultos con epilepsia focal fármacorresistente, tratados en un hospital del Seguro Social del Perú. Material y Métodos: Se revisó la base de datos de la Unidad de Epilepsia de Adultos del Hospital Nacional Edgardo Rebagliati, de junio 2016 a junio 2019, a efecto de identificar pacientes a quienes se había implantados el estimulador. Se registraron las características clínicas y de manejo de la epilepsia (i.e., frecuencia de las crisis y regímenes de antiepilépticos (DAE), previas al implante, a los 3 y 6 meses del mismo y al final del periodo de seguimiento. Igualmente, el cambio porcentual promedio en la frecuencia de crisis en cada periodo, analizándose las tasas de respuesta $\geq 50 \%, \geq 75 \%$ y los cambios en DAE, así como los efectos adversos al final del periodo de seguimiento. Resultados: Cinco pacientes con epilepsia multifocal fármacorresistente recibieron implante de ENV por no ser candidatos a cirugía resectiva de epilepsia. La edad promedio fue de 20,6 años, el promedio de duración del seguimiento fue de 21 meses y el promedio porcentual en la reducción de frecuencia de las crisis a los 3 meses, 6 meses y al final del seguimiento (21 meses) fue de $24 \%, 45 \%$ y $69 \%$, respectivamente. Las tasa de respuesta $\geq 50 \%$ y $\geq 75 \%$, fueron $80 \%$ y $40 \%$. Se evidenció una reducción parcial de DAE en solo 1 paciente. El principal efecto adverso fue la ronquera. Conclusiones: La experiencia con ENV, reportada por primera vez en el Perú, demuestra su eficacia como terapia paliativa y un buen perfil de seguridad en adultos con epilepsia multifocal fármacorresistente. La eficacia es significativamente mayor con la estimulación sostenida a largo plazo.

PALABRAS CLAVE: Estimulador del nervio vago, epilepsia, largo plazo, Perú.

\section{SUMMARY}

Objective: To report the efficacy and long-term safety of the Vagus Nerve Stimulation (VNS), in a series of adult patients with drug-resistant focal epilepsy, treated in a hospital of Peru's Social Security System. Methods: Data of the Adult Epilepsy Unit at Edgardo Rebagliati National Hospital, from June 2016 to June 2019, were reviewed in

1 Unidad de Epilepsia, Departamento de Neurología, Hospital Nacional Edgardo Rebagliati Martins, EsSalud. Lima, Perú.

2 Facultad de Medicina, Universidad Nacional Mayor de San Marcos. Lima, Perú.

3 Servicio de Neurocirugía Funcional, Hospital Nacional Edgardo Rebagliati Martins, EsSalud. Lima, Perú.

4 Facultad de Psicología, Universidad Nacional Mayor de San Marcos. Lima, Perú.

a Médico-Neurólogo; ${ }^{\mathrm{b}}$ Médico-Neurocirujano de Epilepsia; ${ }^{\mathrm{c}}$ Neuropsicóloga. 
order to identify patients who had been implanted with VNS. Frequency of seizures and antiepileptic drugs (AED), prior to implantation with VNS, at 3 and 6 months, and at the end of the follow-up period were recorded, as were the average percentage change in the frequency of seizures, response rates of $\geq 50 \%, \geq 75 \%$, and changes in AED and adverse effects. Results: Five patients with drug-resistant multifocal epilepsy who were implanted with VNS for not being candidates to resective epilepsy surgery were identified. The average age was 20.6 years old. The average duration of follow-up from the implant was 21 months and the average percentage in the reduction of the seizure frequency at 3, and 6 months, and at the end of the follow-up period ( 21 months), was $24 \%, 45 \%$ and $69 \%$, respectively. The response rates of $\geq 50 \%$ and $\geq 75 \%$ were $80 \%$ and $40 \%$. There was a partial reduction of AED in only 1 patient. The main adverse effect was hoarseness. Conclusions: The experience with VNS, reported for the first time in Peru, demonstrates its efficacy as palliative therapy, and good safety profile in adults with drug-resistant multifocal epilepsy. The effectiveness is significantly higher with sustained long-term stimulation.

KEYWORDS: Vagus nerve stimulation, epilepsy, long-term, Peru.

\section{INTRODUCCIÓN}

La prevalencia de la epilepsia fluctúa entre 0,4 a $1 \%$ (1). En Latinoamérica la prevalencia ha sido reportada entre 0,6 y 4,4\% (2). Aproximadamente un $65 \%$ de los pacientes se controlan con fármacos antiepilépticos. Sin embargo alrededor de un $35 \%$ de los casos son resistentes o refractarios a la libertad de crisis a pesar de haber recibido 2 esquemas de fármacos antiepilépticos adecuados, bien tolerados, apropiadamente elegidos, correctamente usados y administrados en monoterapia o en combinación $(3,4)$. Estos pacientes, con epilepsia farmacorresistente, tienen un fuerte impacto negativo psicosocial y en su calidad de vida; se asocian a un incremento en la morbimortalidad y generan elevados costos directos e indirectos a los sistemas de salud $(5,6)$.

La cirugía resectiva de epilepsia, es la mejor alternativa para lograr la libertad de crisis hasta en $80 \%$ de pacientes con epilepsia temporal farmacorresistente y hasta en $60 \%$ de pacientes con epilepsias extratemporales (7-9). Pero no todos los pacientes, tras una evaluación exhaustiva y multidisciplinar (evaluación clínica, evaluación neuropsicológica, Video-electroencefalograma, neuroimágenes estructurales y funcionales) resultan ser candidatos. Estos pacientes, entonces, podrían ser candidatos a tratamientos paliativos, que potencialmente podrían mejorar en forma parcial la frecuencia y severidad de las crisis; a pesar que no permitan la libertad de crisis. Algunos de estos pacientes serán aptos para cirugías paliativas de epilepsia: callosotomía y otras desconexiones. Sin embargo algunos otros no lo serán; entonces podrían ser candidatos a neuroestimulación, dieta cetogénica o uso de Cannabis en síndromes seleccionados $(5,10,11)$. En nuestro país, en la actualidad, no se dispone de experiencia sistemática, ni la logística (dietas tecnificadas) para el uso de dieta cetogénica y tampoco de Cannabidiol (CBD) altamente purificado. Precisamente el CBD purificado al $99 \%$, es el derivado cannabinoide que ha demostrado su eficacia en solo 2 síndromes epilépticos: Lennox Gastaut y Dravet (12).

Desde inicios de este siglo ha habido avances sostenidos y progresivos en el uso clínico de la neuroestimulación en epilepsia. En la actualidad, se encuentran aprobadas por la Administración de Alimentos y Medicamentos (FDA) de los Estados Unidos de Norteamérica (USA) 3 modalidades de neuroestimulación para el uso clínico en pacientes con epilepsia farmacorresistente: estimulación cerebral profunda teniendo como diana al núcleo anterior del tálamo, neuroestimulación cortical responsiva y estimulación del nervio vago $(13,14)$. La estimulación del nervio vago (ENV) es la modalidad más ampliamente usada, investigada y la única disponible en los sistemas de salud pública de la mayoría de países de Latinoamérica. Fue aprobado por la FDA en 1997 para el uso en pacientes mayores de 12 años con epilepsia focal farmacorresistente, en base a un estudio randomizado, controlados y ciego (denominado EO3). Este estudio y un segundo estudio randomizado (denominado EO5), compararon 2 paradigmas de estimulación: terapéutico vs subterapéutico, demostrando una reducción del 25 a $30 \%$ en la frecuencia de crisis a los 3 meses. Posteriores estudios abiertos observacionales y retrospectivos han evidenciado un incremento en la reducción de crisis con el incremento de la duración de la terapia a largo plazo con ENV $(13,15,16)$. En los últimos 3 años se ha implantado estimuladores del nervio vago a un número reducido de pacientes en nuestro país, pero hasta la fecha no se han reportados formalmente los resultados en estos pacientes. 
Eficacia y seguridad a largo plazo de la estimulación del nervio vago: Reporte preliminar en pacientes adultos.

El objetivo del presente trabajo fue reportar la experiencia en eficacia y seguridad a largo plazo de la estimulación del nervio vago en una serie de pacientes adultos con epilepsia multifocal farmacorresistente tratados en La Unidad de Epilepsia del Hospital Rebagliati del Seguro Social del Perú (EsSalud).

\section{MATERIAL Y MÉTODOS}

Estudio descriptivo, retrospectivo en pacientes adultos con epilepsia focal farmacorresistentes implantados con ENV en el Hospital Rebagliati de EsSalud de Lima - Perú. Se revisó la base de datos de La Unidad de Epilepsia de Adultos del Hospital Rebagliati desde junio del 2016 hasta junio del 2019 y se identificó a quienes habían sido implantados con ENV por indicación de la Junta Medica multidisciplinar de La Unidad de Epilepsia de Adultos, luego de cumplir con nuestro protocolo de evaluación para epilepsia farmacorresistente. Este protocolo puede ser consultado en una publicación previa (17). En EsSalud, se atienden en los servicios de adultos a todos los pacientes mayores a 14 años. Se obtuvo información de cada paciente a partir de la base de datos y sus respectivas historias clínicas; sobre el tipo de epilepsia y su etiología, así como la frecuencia de crisis y regímenes de drogas antiepilépticas (DAE), previo al implante con ENV, a los 3 meses, 6 meses del implante y al final del periodo de seguimiento posterior al implante. Se comparó, respecto al basal, el cambio porcentual promedio en la frecuencia de crisis a los 3 meses, 6 meses y hacia el final del periodo de seguimiento. Se registró la tasa de respondedores con una reducción de al menos $50 \%$ de la frecuencia de sus crisis (TR50) y la tasa de respondedores con una reducción de al menos $75 \%$ de la frecuencia de sus crisis (TR75) y los cambios en los regímenes de DAE. Además, se describió los efectos adversos al final del periodo de seguimiento. Para el análisis de los resultados, empleamos estadística descriptiva y para determinar la significancia en el cambio porcentual promedio de la frecuencia de crisis hacia el final del seguimiento respecto a los 3 meses del implante, utilizamos la t de student.

\section{RESULTADOS}

En nuestra base de datos, de 122 pacientes evaluados para posibilidad de cirugía de epilepsia, encontramos 6 pacientes con epilepsia focal farmacorresistente, quienes no fueron candidatos a cirugía resectiva de epilepsia y a quienes se les implantó el estimulador del nervio vago. En los 6 pacientes, el monitoreo prolongado con VideoElectroencefalograma de superficie identificó más de una zona epileptógena (epilepsia multifocal). Para catalogar que el paciente presentaba más de un foco epileptógeno, fue requisito que cada foco epileptógeno tuviese su propia semiología y su propia actividad eléctrica ictal. Debido a los altos costos del ENV, los criterios para decidir el implante en adultos, en nuestro centro los hemos restringido a pacientes con epilepsia multifocal, no candidatos a cirugía resectiva de epilepsia y algunos casos seleccionados en quienes la zona epileptógena (ZE) asiente en una zona elocuente. Por esta razón y al ser la evidencia más débil para epilepsias generalizadas y combinadas refractarias como el síndrome del Lennox Gastaut; en estos casos priorizamos la callosotomía frente al ENV. De los 6 pacientes, se excluyó a una paciente por tener un periodo de implante menor a un mes.

Se analizaron, por tanto, los resultados de 5 pacientes ( 3 mujeres y 2 varones), con una edad promedio de 22,2 \pm 3,7 años (19 -28 años). La edad media de inicio de la epilepsia fue de 7,3 $\pm 8,7$ años (6 meses-21 años) y la edad media al momento del implante fue de 20,6 \pm 4 ,2 años (17 -27 años). Los 5 pacientes se encuentran estimulados con paradigmas de estimulación estándar, con un periodo inicial de rampa de 6 semanas, en el que se incrementó la corriente de salida desde 0,25 miliamperios (mA) hasta 1,5 mA (tabla 1). Las características clínicas y las medidas de la eficacia las describimos en la tabla 2. El promedio de duración del seguimiento desde el implante fue de $21 \pm 3,3$ meses y los promedios de la reducción porcentual de la frecuencia de las crisis a los 3 meses, 6 meses y al final del periodo de seguimiento (promedio de 21 meses) fueron de $24 \%, 45,2 \%$ y

Tabla 1. Parámetros de estimulación del nervio vago.

\begin{tabular}{lllll}
\hline $\begin{array}{l}\text { Corriente de salida } \\
(\mathrm{mA})\end{array}$ & $\begin{array}{l}\text { Frecuencia de estímulo } \\
(\mathrm{Hz})\end{array}$ & $\begin{array}{l}\text { Periodo en On } \\
\text { (segundos) }\end{array}$ & $\begin{array}{l}\text { Periodo en Off } \\
\text { (minutos) }\end{array}$ & $\begin{array}{l}\text { Ancho del pulso } \\
\text { (microsegundos) }\end{array}$ \\
\hline $2-2,5$ & 30 & 30 & 5 & 500 \\
\hline
\end{tabular}


Barreto-Acevedo E, et al.

Tabla 2. Características clínicas y resultado de eficacia en pacientes implantados con ENV

\begin{tabular}{|c|c|c|c|c|c|}
\hline Paciente $\mathrm{N}^{\circ}$ & 1 & 2 & 3 & 4 & 5 \\
\hline Edad (años) & 22 & 19 & 23 & 19 & 28 \\
\hline Sexo & $\mathrm{F}$ & $\mathrm{F}$ & M & M & $\mathrm{F}$ \\
\hline Edad de inicio de epilepsia & 6 meses & 2 años & 2 años & 11 años & 21 años \\
\hline $\begin{array}{l}\text { Edad al momento del } \\
\text { implante (años) }\end{array}$ & 20 & 17 & 22 & 17 & 27 \\
\hline Tipo de epilepsia & $\begin{array}{c}\text { Multifocal } \\
\text { (Bifrontal } \\
\text { independiente) }\end{array}$ & $\begin{array}{l}\text { Multifocal } \\
\text { (Bifrontal } \\
\text { independiente) }\end{array}$ & $\begin{array}{l}\text { Multifocal } \\
\text { (Frontal } \\
\text { derecho y } \\
\text { temporal } \\
\text { izquierdo) }\end{array}$ & $\begin{array}{l}\text { Multifocal } \\
\text { (Frontal } \\
\text { derecho y } \\
\text { temporal } \\
\text { izquierdo) }\end{array}$ & $\begin{array}{c}\text { Multifocal } \\
\text { (Bitemporal } \\
\text { con focos } \\
\text { independientes) }\end{array}$ \\
\hline Diagnóstico etiológico & $\begin{array}{l}\text { Esclerosis } \\
\text { Tuberosa }\end{array}$ & $\begin{array}{l}\text { Doble corteza } \\
\text { y Paquigiria } \\
\text { bifrontal }\end{array}$ & Desconocido & Desconocido & $\begin{array}{c}\text { Esclerosis } \\
\text { Mesial Temporal } \\
\text { bilateral }\end{array}$ \\
\hline $\begin{array}{l}\text { Periodo de seguimiento } \\
\text { (meses) }\end{array}$ & 26 meses & 22 meses & 19 meses & 17 meses & 21 meses \\
\hline $\begin{array}{l}\text { Reducción porcentual de } \\
\text { crisis respecto al basal a los } \\
3 \text { meses }\end{array}$ & $0 \%$ & $10 \%$ & $40 \%$ & $40 \%$ & $30 \%$ \\
\hline $\begin{array}{l}\text { Reducción porcentual de } \\
\text { crisis respecto al basal a los } \\
6 \text { meses }\end{array}$ & $20 \%$ & $34 \%$ & $65 \%$ & $55 \%$ & $52 \%$ \\
\hline $\begin{array}{l}\text { Reducción porcentual de } \\
\text { crisis respecto al basal al } \\
\text { final del seguimiento }\end{array}$ & $48 \%$ & $82 \%$ & $80 \%$ & $70 \%$ & $68 \%$ \\
\hline $\begin{array}{l}\text { Esquema DAE pre- } \\
\text { implante }\end{array}$ & $\begin{array}{c}\mathrm{LEV} 3 \mathrm{~g} / \mathrm{d} \\
\mathrm{CBZ} 800 \mathrm{mg} / \mathrm{d} \\
\text { VPA2 } \mathrm{g} / \mathrm{d}\end{array}$ & $\begin{array}{l}\text { LEV } 3 \mathrm{~g} / \mathrm{d} \\
\text { LTG } 0,5 \mathrm{~g} / \mathrm{d} \\
\text { VPA } 1 \mathrm{~g} / \mathrm{d}\end{array}$ & $\begin{array}{c}\text { CZP } 8 \mathrm{mg} / \mathrm{d} \\
\text { TPM } 0,3 \mathrm{~g} / \mathrm{d} \\
\text { VPA } 1,5 \mathrm{~g} / \mathrm{d} \\
\text { PB } 125 \mathrm{mg} / \mathrm{d}\end{array}$ & $\begin{array}{c}\mathrm{LEV} \mathrm{3g/d} \\
\mathrm{LTG} 300 \mathrm{mg} / \mathrm{d} \\
\text { CLB 30mg/d }\end{array}$ & $\begin{array}{c}\text { LEV } 3 \mathrm{~g} / \mathrm{d} \\
\mathrm{LTG} 500 \mathrm{mg} / \mathrm{d} \\
\text { CZP } 1 \mathrm{mg} / \mathrm{d}\end{array}$ \\
\hline $\begin{array}{l}\text { Cambio DAE al final del } \\
\text { seguimiento }\end{array}$ & Ninguno & Ninguno & $\begin{array}{c}\text { Suprimió CZP. } \\
\text { Redujo PB a } \\
75 \mathrm{mg} / \mathrm{d}\end{array}$ & Ninguno & Ninguno \\
\hline
\end{tabular}

LEV: Levetiracetam. CBZ: Carbamazepina. VPA:Acido Valproico. LTG:Lamotrigina. CZP:Clonazepam. TPM: Topiramato. PB: Fenobarbital. CLB: Clobazam. g/d: gramos/día mg/d: miligramos/día.

Tabla 3. Efectos adversos en pacientes implantados con ENV

\begin{tabular}{lc}
\hline Efecto adverso & $\mathrm{N}^{\circ}$ de pacientes \\
\hline Ronquera & 4 \\
Tos & 2 \\
Parestesias cervical o faríngeo & 1 \\
Dolor cervical o faríngeo & 0 \\
Disnea & 0 \\
Cefalea & 0 \\
Parálisis de cuerda vocal & 0 \\
Infecciones o seromas o hematomas & 0 \\
\hline
\end{tabular}


Eficacia y seguridad a largo plazo de la estimulación del nervio vago: Reporte preliminar en pacientes adultos.

$69 \%$. Hubo una reducción significativa $(\mathrm{p}<0.05)$ en la frecuencia de crisis al final del seguimiento respecto a los 3 meses del implante; en tanto que la mejoría fue modesta y no homogénea a los 3 meses. Hacia el final del periodo de seguimiento, la tasa de respondedores TR50 y TR75 fue de $80 \%$ y $40 \%$, respectivamente. Se evidenció una reducción parcial de los DAE en solo 1 paciente, sin registrarse cambios en la dosis o en el esquema de DAE de los otros pacientes. El principal efecto adverso fue la ronquera y no hubo eventos adversos serios (tabla 3 ).

\section{DISCUSIÓN}

La eficacia de la neuroestimulación con ENV en nuestra serie de pacientes adultos con epilepsia farmacorresistente es concordante con los resultados reportados en la literatura mundial. En los primeros 3 meses de seguimiento, las tasas porcentuales de reducción de crisis, no fueron homogéneas en nuestros 5 pacientes y el promedio porcentual en la reducción de crisis fue modesto (24\%), aunque se encuentra dentro de lo reportado. Así los estudios EO3 y EO5, primeros estudios controlados, randomizados y ciegos en evaluar la eficacia del ENV encontraron tasas similares luego en un periodo de seguimiento de alrededor de 3 meses $(15,16)$. En el estudio EO3, multicéntrico (17 centros) con 114 pacientes con epilepsia focal farmacorresistente, mayores de 12 años; se comparó la eficacia en cuanto a reducción porcentual en la frecuencia de crisis respecto al basal pre-implante en 2 paradigmas de estimulación: terapéutico vs presumiblemente subterapéutico y se encontró una cifra de $25 \%$ para el primer grupo (15). En el estudio EO5, también multicéntrico (20 centros) en 196 pacientes mayores de 12 años y con un diseño similar; la tasa de reducción porcentual en la frecuencia de crisis respecto al basal fue de $28 \%$ para el grupo de estimulación terapéutica (16). En ambos estudios se encontró diferencia significativa en comparación al paradigma presumiblemente subterapéutico. Las debilidades de ambos estudios son: haber sido auspiciados por el fabricante y la no utilización de un verdadero placebo; pero ambos aspectos no invalidan sus resultados y precisamente la FDA se basó en el primero de ellos para aprobar la utilización del ENV en USA (13).

En los estudios EO3 y EO5, la tasa de respondedores $\geq 50 \%$ (TR50), a corto plazo (3 meses) fueron de $31 \%$ y $23.4 \%$ respectivamente para los grupos en estimulación terapéutica; pero solo en el estudio EO3 se encontró diferencia significativa respecto al grupo subterapéutico. En tanto que en el estudio EO5, si se encontró diferencia significativa en la tasa de respondedores $\geq 75 \%$ (TR75) $(10.6 \%$ vs $2 \%$, grupo terapéutico vs subterapéutico) $(15,16)$. En nuestro estudio hemos encontrado una tasa más elevada de respondedores al final del periodo de seguimiento (TR50 de $80 \%$ y TR75 de 40\%), esto podría explicarse por la reducida muestra de nuestro estudio y por el mayor periodo de seguimiento que los estudios EO3 y EO5. De manera similar a nuestro estudio Amar et al, en 1998, publicaron un estudio controlado y randomizado con bajo número de pacientes (7 con estimulación terapéutica y 10 con estimulación subterapéutica) y encontraron tasas elevadas en: reducción porcentual de crisis a los 3 meses para el grupo con estimulación terapéutica ( $71 \%$ vs $6 \%$ ) y en la tasa de respondedores $\geq 75 \%$ ( $77 \%$ vs $10 \%)$ (18).

Cuando analizamos las tasas porcentuales de la reducción en la frecuencia de crisis a lo largo del tiempo, observamos que se incrementa la mejoría con el incremento del periodo de seguimiento y las cifras ya no son tan heterogéneas en los 5 pacientes como lo eran a los 3 meses del seguimiento. De esta forma, a los 6 meses de seguimiento la cifra promedio de reducción porcentual en la frecuencia de crisis se incrementó a $45 \%$ y al final del periodo de seguimiento (promedio 21 meses) a un 76\%. Esta mejoría increscendo con el tiempo de uso del ENV, ha sido también evidenciada en varios estudios de seguimiento observacionales prospectivos, estudios retrospectivos y estudios en base a registros de pacientes, hasta por periodos mayores a 10 años de seguimiento. Amar et al, realizaron el seguimiento por 15 meses a 164 pacientes del estudio EO5, en el que a los pacientes del grupo de estimulación subterapéutica se les mutó a estimulación con parámetros terapéuticos. Encontraron un incremento en las medidas de eficacia respecto a lo encontrado a los 3 meses por el estudio EO5: la tasa de reducción porcentual promedio subió a 45\%; y las tasas de respondedores TR50 y TR75 se elevaron a $39 \%$ y $21 \%$, respectivamente (19). En la misma línea, DeGiorgio et al, realizaron un seguimiento de pacientes del estudio EO5 $(n=195)$ y encontraron un incremento significativo $(p<0,001)$ en el tiempo a los 15 meses vs a los 3 meses del término del estudio EO5 para las siguientes medidas de eficacia: TR75 $16 \%$ a los 3 meses y $20 \%$ a los 15 meses; reducción porcentual promedio en las crisis, de $34 \%$ a los 3 meses a $45 \%$ a los 15 meses (20).

Morris $\mathrm{G}$ et al, en un estudio prospectivo, de etiqueta abierta, en 454 pacientes, también documentó 
un incremento de la eficacia en el tiempo: la TR50 se elevó de $36.8 \%$ al año de seguimiento hasta $43 \%$ al segundo año y la reducción porcentual en la frecuencia de la crisis se incrementó de $35 \%$ al año de seguimiento a $44 \%$ a los 2 años de seguimiento (21). García - Navarrete et al, en un estudio español, también prospectivo y de etiqueta abierta, en 43 pacientes con epilepsia farmacorresistente, con edad promedio de 34 años y sin cambio en las DAE durante el periodo de seguimiento, encontró una TR50 de 63\% y una reducción porcentual promedio de $46.6 \%$ a los 18 meses de seguimiento (22). Vonck et al, en 118 pacientes con edad promedio de 32 años, seguidos por 33 meses, encontraron una TR50 de 51\% y una tasa de reducción porcentual promedio de $56 \%$ para "crisis parciales complejas", 64\% para "crisis parciales simples" y 49\% para "crisis con generalización" (23). Ardesh et al., en un estudio holandés con 19 pacientes adultos, también encontraron incrementos con el tiempo en las medidas de eficacia del ENV: la TR50 paso de $12 \%$ al año de seguimiento a $38 \%$ al quinto año y la tasa de reducción porcentual promedio en la frecuencia de crisis mejoró de $14 \%$ en el primer año a $50 \%$ al quinto año (24).

En tres estudios en base a registro prospectivo de un gran número pacientes en múltiples centros, también se corroboró la mejoría increscendo de la eficacia del ENV. El estudio de Englot con 5554 pacientes, con edad promedio de 27 años, evidenció que la TR50 pasó de $49 \%$ a los 4 meses de seguimiento, a $55 \%$ a los 24 meses y a $63 \%$ a los 48 meses; además se registró un $47 \%$ de reducción porcentual promedio en la frecuencia de crisis a los 4 meses (25). En el estudio de Labar con 269 pacientes registrados, con edad promedio de 32 años y sin cambio en las DAE durante el seguimiento, se evidenció un incremento en la TR50 de $48 \%$ a los 3 meses, a $57 \%$ a los 12 meses y un incremento en la TR75 de $23 \%$ a los 3 meses, a $35 \%$ a los 12 meses. La tasa de reducción porcentual promedio en la frecuencia de crisis también se incrementó significativamente del $45 \%$ a los 3 meses al $58 \%$ a los 12 meses (26). El estudio de registro de Amar et al., incluyó a 2 subgrupos (921 operados de epilepsia y 3822 no operados previamente), la mejoría con el tiempo se incrementó en ambos grupos: la TR50 de $48 \%$ y $50 \%$ en los operados y no operados a los 12 meses, se elevó a 55\% y $62 \%$ a los 24 meses respectivamente. Así mismo la reducción porcentual promedio en la frecuencia de las crisis se incrementó de $43 \%$ y $47 \%$ en operados y no operados a los 3 meses, a $46 \%$ y $60 \%$ a los 6 meses; y a $51 \%$ y $67 \%$, a los 24 meses, respectivamente (27).
Los estudios con mayor periodo de seguimiento son retrospectivos, y también corroboran la mejoría de la eficacia con el tiempo. El estudio de Spanaki et al, con 26 pacientes y sin cambios significativos en la carga de DAE, evidenció un incremento en la reducción porcentual promedio de la frecuencia de crisis: de $28 \%$ al año de seguimiento a $72 \%$ a los 5 años (28). Kuba et al., en un estudio checo, encontraron un incremento en la TR50 con el tiempo, de 44\% al año de seguimiento a $64 \%$ a los 5 años; además evidenció un reducción promedio de $56 \%$ en la frecuencia de crisis al finalizar el seguimiento (29). Elliot et al., en un primer estudio con 65 pacientes y seguimiento hasta por más de 10 años, encontraron un incremento progresivo en la reducción porcentual promedio de la frecuencia de crisis en función al tiempo de uso del ENV: $35,7 \%$ a los 6 meses de seguimiento, $52,1 \%$ al año, $58,3 \%$ a los 2 años, $60,4 \%$ a los 4 años, $65,7 \%$ a $\operatorname{los} 6$ años, $75 \%$ a los 8 años y 76,3\% pasado los 10 años de seguimiento; además encontraron un TR50 de $90,8 \%$ y una TR75 de $58,5 \%$ pasado los 10 años (30). Elliot et al., replicaron el trabajo en 436 pacientes con seguimiento de hasta casi 5 años y encontró una TR50 de 64\% y una TR75 de 40,5\%, en tanto que la reducción porcentual promedio en la frecuencia de crisis fue de 55,8\% (31); estos estudios restrospectivos tienen algunas limitaciones metodológicas además de su diseño (sesgos potenciales en la recopilación de los datos e imprecisión en el seguimiento cuando se maneja grandes muestras); como el incluir a poblaciones no homogéneas, pues incluyen a niños y adultos y a pacientes con epilepsias focales y generalizadas.

En el 2014, Ryvlin publicó un ensayo clínico controlado, a etiqueta abierta, no ciego que comparó la eficacia en mejoría de calidad de vida, medida por el cuestionario QOLIE89, en 2 grupos: DAE+ENV vs DAE y encontró una mejoría estadísticamente significativa en la calidad de vida a favor del grupo de ENV+DAE. Este estudio tuvo además como objetivo secundario establecer cambios en la TR50 y cambios porcentuales en la frecuencia de crisis. Aun cuando hubo mejoría significativa en la calidad de vida y una mayor reducción porcentual en la frecuencia de las crisis a lo largo del seguimiento (12 meses) en el grupo de ENV+DAE, esta no fue significativa respecto al grupo control y tampoco lo fue la TR50. Sin embargo cuando se comparó la reducción de la frecuencia absoluta de crisis semanal, esta si fue significativa para el grupo ENV+DAE respecto al grupo control al final del periodo de seguimiento (32). 
Eficacia y seguridad a largo plazo de la estimulación del nervio vago: Reporte preliminar en pacientes adultos.

Hasta la actualidad no hay una explicación certera, de porque la neuroestimulación sostenida con el tiempo y a largo plazo del nervio vago mejora su eficacia. Se ha propuesto que un mayor tiempo para la neuroestimulación permitiría una más óptima titulación de los parámetros de estimulación pues estos se modifican gradualmente. Es probable, además, que un mayor tiempo de neuroestimulacion favorecería una más óptima remodelación o modulación de redes o circuitos neuronales inhibitorios. Explicar la mejoría en el tiempo por el fenómeno de regresión a la media o la fluctuación natural de la frecuencia en las crisis de un grupo de pacientes con epilepsia es menos factible, debido a que los periodos de seguimiento son bastante prolongados. Atribuir la mejoría a la optimización en los esquemas de las DAE mientras los pacientes están expuestos a ENV, tampoco es una explicación del todo satisfactoria, debido a que gran parte de los estudios de seguimiento a mediano y largo plazo no han evidenciado modificaciones significativas en la carga o esquemas de DAE, como ocurrió en nuestro estudio; sin embargo un grupo de pacientes podría beneficiarse de la sinergia de la optimización de los parámetros de estimulación y a la vez de la optimización de los esquemas de los DAE en periodos prolongados. Finalmente la eficacia clínica con el tiempo, debería también ser entendida no solo como la mejoría en el control de crisis, sino de otros parámetros (ejemplo: estado de alerta, estado de ánimo, cognición) que en global mejoran la calidad de vida como lo demuestra el estudio de Ryvlin (22, 26, 28, 30-32).

Respecto a los efectos adversos, de la misma forma que hemos encontrado en nuestra serie, los estudios de seguimiento a corto y largo plazo, coinciden en un adecuado perfil de seguridad del ENV. Los riesgos de este procedimiento, que es poco invasivo y reversible; son habitualmente menores, autolimitados (fenómeno de habituación), bien tolerados por el paciente, no requieren modificaciones significativas en los parámetros de estimulación y no son causal de retiro del dispositivo en la gran mayoría de casos. En forma homogénea y de forma similar a nuestros resultados, el efecto adverso más frecuente encontrado en otros estudios, es el cambio en el tono de voz (ronquera o disfonía). Este se reporta entre un 37 a $84 \%$ de los pacientes implantados y en los estudios EO3 y EO5 fue significativamente mayor en el grupo de estimulación terapéutica que el grupo con estimulación subterapéutica. Otros eventos adversos de escasa severidad, incluyen a la tos y parestesias cervicales o faríngeas que las evidenciamos en tres de nuestros pacientes y fueron transitorias al iniciar el periodo de rampa y solo durante la estimulación (periodos "on"). La tos ha sido reportada en 11 a $45 \%$ de pacientes y las parestesias en 6 a $25 \%$. En nuestro estudio no encontramos otros efectos adversos menores, ni tampoco efectos adversos serios como parálisis de cuerda vocal, fractura del cable del electrodo, hematomas, seromas o infecciones en el entorno del estimulador. En otros estudios se han reportado disnea entre 6 a $25 \%$, cefalea entre 5 a $24 \%$, náuseas entre 7 a $20 \%$ y dolor cervical - faríngeo entre 11 a $25 \%$. Son raras las complicaciones serias antes referidas, que ocasionalmente pueden obligar a que los generadores sean inactivados transitoriamente $\mathrm{o}$ explantados $(15,16,18,20,22,24,29,31)$

A pesar de la evidencia de eficacia del ENV como terapia paliativa en pacientes adultos con epilepsia refractaria y su buen perfil de seguridad a largo plazo; la principal limitante es el costo de los dispositivos, que en nuestro país bordean los 36000,00 dólares. En países desarrollados se han realizado algunos estudios de costos con resultados favorables para el ENV (33-37), sin embargo no se disponen de estos estudios en países en vías de desarrollo, como los latinoamericanos. Otra limitante del ENV es que a pesar que se ha investigado sobre factores predictores de buena respuesta al ENV para orientar a una mejor selección de pacientes, los resultados no han sido homogéneos y los estudios al respecto han tenido limitaciones metodológicas (retrospectivos o estudios en base a registros). Entre los factores predictores de buena respuesta estudiados encontramos: mayor edad (>12 años), corta duración de la epilepsia previo al implante, epilepsias focales o multifocales, epilepsias no lesionales y entre las epilepsias con etiologías conocidas: mejor respuesta en epilepsias postinfecciosas que en las debidas a malformaciones corticales y habría una respuesta menos favorable en desordenes de la migración neuronal. Nuestros pacientes han presentado algunas de estas características: mayores de 12 años, epilepsias multifocales, 2 pacientes con epilepsia sin lesión aparente en la resonancia de encéfalo. Sin embargo no todos los estudios encuentran homogéneamente estos resultados y los factores señalados deben ser tomados con cautela $(25,26,29,31,38)$

En conclusión, nuestra experiencia con estimulación del nervio vago, reportada por primera vez en el Perú en esta pequeña serie, demuestra su eficacia como terapia paliativa y un buen perfil de seguridad en adultos con epilepsia multifocal refractaria tratados a largo plazo en el Seguro Social. La eficacia es significativamente mayor con la estimulación sostenida a largo plazo. 
Se requiere de estudios de costo- efectividad y costooportunidad en países en vías de desarrollo y de estudios con un mayor número de pacientes en nuestro medio.

Los autores no han recibido financiamiento de ninguna institución para esta investigación. El trabajo ha sido autofinanciado. La Dra. Alicia Becerra Zegarra, recibió auspicio de AxonPharma (representante de Livanova en Perú) para asistir a un curso de capacitación sobre estimulador del nervio vago en U.S.A. Los otros autores declaran no tener conflicto de interés.

\section{Correspondencia}

Dr. Elliot Barreto Acevedo

Hospital Nacional Edgardo Rebagliati Martins

Av. Rebagliati 490 Jesús María

Teléfono: 511-265-4901 anexo: 3081. Celular: 989963057.

Correo electrónico: elliotba@hotmail.com

\section{REFERENCIAS BIBLIOGRÁFICAS}

1. Sander J. The epidemiology of epilepsy revisited. Curr Opin Neurol. 2003; 16 (2):165-170.

2. Burneo G, Tellez-Zenteno J, Wiebe S. Understanding the burden of epilepsy in Latin America: A systematic review of its prevalence and incidence. Epilepsy Research. 2005; 66: 63-74.

3. Kwan P, Arzimanoglou A, Berg A, Brodie M, Hauser A, Mathern G, et al. Definition of drug resistant epilepsy: Consensus proposal by the ad hoc Task Force of the ILAE Commission on Therapeutic Strategies. Epilepsia. 2010; 51:1069-1077.

4. Engel J, Wiebe S, French J, Sperling M, Williamson P, Spencer D, et al. Practice parameter: Temporal lobe and localized neocortical resections for epilepsy. Report of the Quality Standards Subcommittee of the American Academy of Neurology, in Association with the American Epilepsy Society and the American Association of Neurological Surgeons. Neurology. 2003; 60:538-547.

5. Ontario Health Technology Advisory Committee. Recommendation: Care for Drug-Refractory Epilepsy in Ontario.Ontario: Health Quality Ontario; 2012 (Citado el 21 de Octubre 2016) Disponible en: http:// www.hqontario.ca/Portals/0/Documents/evidence/ reports/EpilepsyOHTACRec2012.pdf

6. Pérez P. Costo médico directo de la epilepsia en la población hospitalaria del hospital III Miguel Grau de EsSalud. Acta Med Per. 2010. 27(1): 37- 42.

7. Wiebe S, Blume W, Girvin J, Eliasziw M. A randomized, controlled trial of surgery for temporal lobe epilepsy. N Engl J Med. 2001; 345 (5): 311-18.

8. Engel J, McDermott M, Wiebe S, Langfitt J, Stern J, Dewar $\mathrm{S}$, et al. Early surgical therapy for drug-resistant temporal lobe epilepsy. JAMA. 2012; 307(9):922-30.

9. Téllez-Zenteno J, Hernández L, Moien-Afshari F, Wiebe S. Surgical outcomes in lesional and nonlesional epilepsy: A systematic review and metaanalysis. Epilepsy Res. 2010; 89(2): 310-318.

10. Ryvlin P, Rheims S. Epilepsy surgery: eligibility criteria and presurgical evaluation. Dialogues Clin Neurosci. 2008; 10:91-103.

11. Jobst B. Treatment algorithms in refractory partial epilepsy. Epilepsia. 2009; 50(8): 51-56.

12. Perucca E. Cannabinoids in the Treatment of Epilepsy: Hard Evidence at Last?. Journal of Epilepsy Research. 2017; 7(2):62-77.

13. Nune G, DeGiorgio C, Heck Ch. Nueromodulation in the treatment of epilepsy. Curr Treat Options Neurol. 2015;17:43-8.

14. Chang B. Deep brain stimulation works for drugresistant epilepsy, but how? Epilepsy Currents. 2018; 18 (6): 378-9.

15. The Vagus Nerve Stimulation Study Group. A randomized controlled trial of chronic vagus nerve stimulation for treatment of medically intractable seizures. Neurology.1995: 45(2):224-30.

16. Handforth A, DeGiorgio C, Schachter SC, Uthman BM, Naritoku DK, Tecoma ES, et al. Vagus nerve stimulation therapy for partial-onset seizures: a randomized active-control trial. Neurology. 1998; 51(1):48-55.

17. Barreto-Acevedo E, Villafuerte-Espinoza M, BecerraZegarra A, Diaz-Vasquez A, Hernandez-Vizarreta $\mathrm{J}$, Llaja-Rojas V. Cirugía resectiva de epilepsia lesional focal: Estudio en pacientes adultos del Seguro Social del Perú. Rev Neuropsiquiatr. 2017; 80 (1): 12-21.

18. Amar AP, Heck CN, Levy ML, Smith T, DeGiorgio $\mathrm{CM}$, Oviedo S, Apuzzo ML. An institutional experience with cervical vagus nerve trunk stimulation for medically refractory epilepsy: rationale, technique and outcome. Neurosurgery. 1998; 43(6);1265-76.

19. Amar AP, DeGiorgio C, Tarver WB, Apuzzo ML. Long-term multicenters experience with vagus nerve stimulation for intractable partial seizures: results of the XE5 trial. Stereotact Funct Neurosurg. 1999; 73(1):104-8.

20. DeGiorgio C, Schachter SC, Handforth A, Salinsky M, Thompson J, Uthman B, et al. Prospective longterm study of vagus nerve stimulation for the treatment of refractory seizures. Epilepsia. 2000; 41(9):11951200.

21. Morris GL, Mueller WM. Long-term treatment with vagus nerve stimulation in patients with refractory epilepsy: The Vagus Nerve Stimulation 
Eficacia y seguridad a largo plazo de la estimulación del nervio vago: Reporte preliminar en pacientes adultos.

Study Group E01- E05. Neurology.1999; 53 (8):1731-35.

22. Garcia-Navarrete E, Torres CV, Navas M, Pastor J, Solas RG. Long-term results of vagal nerve stimulation for adults with medication-resistant epilepsy who have been on unchanged antiepileptic medication. Seizure. 2013;22(1):9-13.

23. Ardesch JJ, Buschman HP, Wagener-Schimmel LJ, van der Aa HE, Hageman G.. Vagus nerve stimulation for medically refractory epilepsy: a long-term followup study. Seizure. 2007; 16(7): 579-85.

24. Vonck K, Thadani V, Gilbert K, Dedeurwaerdere S, De Groote L, De Herdt V, et al. Vagus nerve stimulation for refractory epilepsy: a transatlantic experience. J Clin Neurophysiol. 2004; 21(4):283-289.

25. Englot, D, Rolston J, Wright C, Hassnain K, Chang E. Rates and predictors of seizure freedom with vagus nerve stimulation for intractable epilepsy. Neurosurgery. 2015; 1:1-9.

26. Labar DB. Vagus nerve stimulation for 1 year in 269 patients on unchanged antiepileptic Drugs. Seizure. 2004; 13: 392-8.

27. Amar AP, Apuzzo ML, Liu CY. Vagus nerve stimulation therapy after failed cranial surgery for intractable epilepsy: results from the vagus nerve stimulation therapy patient outcome registry. Neurosurgery. 2004, 55(41):1086-1093.

28. Spanaki MV, Allen LS, Mueller WM, Morris GL. Vagus nerve stimulation therapy: 5-year or greater outcome at a university-based epilepsy center. Seizure. 2004; 13(8):587-90.

29. Kuba R, Brázdil M, Kalina M, Prochazka T, Hovorka J, Neza T, et al. Vagus nerve stimulation: longitudinal follow-up of patients treated for 5 years. Seizure. 2009; 18(4):269- 74.

30. Elliott RE, Morsi A, Tanweer O. Efficacy of vagus nerve stimulation over time: review of 65 consecutive patients with treatment-resistant epilepsy treated with VNS>10 years. Epilepsy Behav. 2011; 20(3):478-83.
31. Elliott RE, Morsi A, Kalhorn SP, Marcus J, Sellin J, Kang M, Grobelny B, Getler E, Carlson Ch, et al. Vagus nerve stimulation in 436 consecutive patients with treatment-resistant epilepsy: long-term outcomes and predictors of response. Epilepsy Behav. 2011;20 (1):57-63.

32. Ryvlin P, Gilliam F, Nguyen D, Colicchio G, Ludice A, Tinuper $\mathrm{P}$, et al. The long-term effect of vagus nerve stimulation on quality of life in patients with pharmacoresistant focal epilepsy: The PuLsE (Open Prospective Randomized Long-term Effectiveness) trial. Epilepsia. 2014; 55(6):893-900.

33. Ben-Menachem E, Hellstrom K, Verstappen D. Analysis of direct hospital costs before and 18 months after treatment with vagus nerve stimulation therapy in 43 patients. Neurology. 2002;59(4):S44-7.

34. Boon P, D'Have M, Van Walleghem P. Direct medical costs of refractory epilepsy incurred by three different treatment modalities: a prospective assessment. Epilepsia. 2002;43: 96-102.

35. Forbes RB, Macdonald S, Eljamel S, Roberts RC. Cost-utility analysis of vagus nerve stimulators for adults with medically refractory epilepsy. Seizure. 2003;12:249-56.

36. Helmers S, Sheng M, Guérin A, Sarda S, Samuelson $\mathrm{T}$, Bunker M, et al. Clinical and economic impact of vagus nerve stimulation therapy in patients with drugresistant epilepsy. Epilepsy \& Behavior. 2011; 22: $370-5$.

37. Camp C, Smithson W, Bunker M, Burke T, Hughes D. Impact of vagus nerve stimulation on secondary care burden in children and adults with epilepsy: Review of routinely collected hospital data in England. Epilepsy \& Behavior. 2015; 52: 68-73.

38. Boon P, De Cock E, Mertens A, Trinka E. Neurostimulation for drug-resistant epilepsy: a systematic review of clinical evidence for efficacy, safety, contraindications and predictors for response. Curr Opin Neurol. 2018, 31: 1-13

Recibido: 10/08/2019

Aceptado: 12/09/2019 